\title{
A detector for the sources
}

Henry N. Chapman

A new detector built for $\mathrm{X}$-ray free-electron lasers provides unprecedented speed and accuracy for macromolecular crystallography at synchrotron radiation facilities - and finally allows crystallographers to harness the full capabilities of those sources.

The development of synchrotron radiation sources, used for applications ranging from chemistry, physics, to materials, environmental and life sciences, has been one of exponential growth, outpacing even Moore's law. Despite large resources devoted to improving source brightness with each new facility and facility upgrade, measurement capabilities rarely can take full advantage of these due to a significant lag in capable detectors. With the newly-developed JUNGFRAU detector from the Paul Scherrer Institute (PSI), this gap has at last been closed, at least for the application of macromolecular crystallography. Ironically, this only happened due to even more demanding sources -X-ray free-electron lasers (XFELs). Built for use at the SwissFEL source at PSI, Leonarski et al took this two-dimensional pixel array detector to a synchrotron. As reported on $\mathrm{p}$. ??, they showed it can record data at a rate matching the flux of photons diffracting from a protein crystal at a modern beamline 1 . A full dataset was obtained in $0.6 \mathrm{~s}$, from a crystal rotating at 100 degrees per second. The new technology has a surprising additional benefit that it gives crystallography data of higher accuracy and quality than of the widely used hybrid photon counting (HPC) detectors, such as the PILATUS and EIGER detectors manufactured by DECTRIS. The JUNGFRAU detector promises significant improvements in data collection for macromolecular structure determination as needed for native phasing (using weak anomalous diffraction from sulphur atoms in the protein) and meets the requirements for rapid data collection at exposure times down to single $\mathrm{X}$-ray pulses at the next generation of diffraction-limited storage ring facilities.

The high energy of X-ray photons makes them easy to detect. Indeed, X-rays were discovered by Röntgen by their accidental exposure of photographic film-an example of an integrating detector. Early accurate measurements were achieved by counting visible scintillations in a phosphor, converted by a photomultiplier tube to current pulses (giving audible clicks if connected to a loudspeaker). Applications such as macromolecular crystallography require the measurement of the intensity and distribution of diffracted intensities from a sample. Although less quantitative, film was the practical choice since it resolves the pattern of the Bragg spots of a protein crystal, to measure them without excessive radiation exposure. Digital two-dimensional detectors followed, including image plates (integrating), CCDs coupled to a scintillator (integrating), and the HPC detectors mentioned above. The latter currently dominate X-ray scattering instruments at synchrotron sources due to their low noise and high frame rates. The most recent HPC detector - the EIGER - can count at a rate exceeding $10^{6}$ photons/s per pixel.

Photon counting detectors are not so useful at XFELs - all photons arrive at a detector pixel within the femtosecond duration of an X-ray pulse, giving just one current spike to count. Several integrating detectors have thus been developed ${ }^{2,3}$. The JUNGFRAU detector overcomes the slow readout of CCDs with addressable pixels of HPC detectors. The electronics in each pixel (see figure) can independently switch its gain setting-providing a dynamic range of $10^{4}$, a requirement for XFEL single-shot measurements. These characteristics mean that at more steady 
synchrotron source $2.4 \times 10^{7}$ photons can be counted per pixel per second, a rate that exceeds that of the EIGER, for Leonarski's and colleagues' feat of a full measurement in $0.6 \mathrm{~s}$. Another detector originating from the same technology base crams many analogue storage cells into each pixel, allowing bursts of 352 frames with as little as $200 \mathrm{~ns}$ separation (a $5 \mathrm{MHz}$ frame rate). Built for the even greater demands of the European XFEL, the high dynamic range of this Adaptive Gain Integrating Pixel Detector was demonstrated by measuring a small-angle X-ray scattering (SAXS) pattern without a beamstop, measuring simultaneously the direct synchrotron beam and single photons ${ }^{3}$.

A benefit of charge integration is that the pixels have a uniform response to X-rays, which turns out to be necessary for accurate measurements. A counting detector only gives a count if the current spike exceeds a threshold-the charge cloud from a photon impacting near pixel boundaries will be split between them and might be missed. An integrating detector will not lose photons. Leonarski et al mapped out the pixel responses in the JUNGFRAU and EIGER detectors using the crystal Bragg spots themselves, and found significant variations in the latter case. They show this explains an observed improvement in the anomalous diffraction signal from native sulphur atoms. Sulphur single-wavelength anomalous diffraction, used to obtain a non-biased structure solution, has the most stringent demands on accuracy, relying on an effect of 0.24 electrons per $S$ atom. Two to three times more data were required with the EIGER detector to obtain similar performance. As a tip to those unlucky not to have a JUNGFRAU (there are no plans yet to commercialise it), dithering the detector position by sub-pixel amounts can improve data accuracy!

The high speed of the JUNGFRAU detector bodes well for the next generation of diffractionlimited storage ring sources and facility upgrades ${ }^{4}$. Their increased brightness means that highly focused and intense beams can be directed onto micrometer-sized protein crystals. The method of serial crystallography-likewise developed first for XFELs - takes full advantage of such high intensities by streaming crystals across the beam, with microsecond or shorter exposures for low dose measurements. The JUNGFRAU detector would allow over 1000 crystals to be measured per second, opening up large ensemble measurements of triggered reactions or responses of proteins to varied conditions. Even with today's sources, measurements could be made hundreds of times faster than the blazing speed of Leonarki's demonstration (down to exposures of single X-ray pulses) by discarding the X-ray monochromator to use the so-called "pink" beam".

The JUNGFRAU and other XFEL detectors show that it is possible to build detectors for future capabilities of synchrotron facilities, rather than current requirements, although this was somewhat unintentional. The detector deficit at synchrotrons is sometimes compared with highenergy physics, where about $10 \%$ of construction costs are devoted to the detector. The heterogeneity of synchrotron research complicates such coordination, but only a few detector types address most applications. Like in particle physics, the ultimate detector would resolve the energy and momentum (i.e. scattering angle) of every photon scattered from or emitted by the sample, tagged in time. One thing is clear: detector requirements for synchrotron and FEL facilities will continue to grow, and addressing the greatest challenges benefits instruments at all sources. 
1. F. Leonarski et al., "Fast and Accurate Data Collection for Macromolecular Crystallography Using the JUNGFRAU Detector," Nature Meth. ??

2. H. T. Philipp, L. J. Koerner, M. S. Hromalik, M. W. Tate, and S. M. Gruner, "Femtosecond radiation experiment detector for $x$-ray free-electron laser (XFEL) coherent $x$-ray imaging," IEEE Trans. Nucl. Sci. 57, 3795-3799 (2010).

3. A. Allahgholi et al. "AGIPD, a high dynamic range fast detector for the European XFEL," J. Instrum. 10, C01023 (2015).

4. M. Eriksson, J. F. van der Veen, and C. Quitmann, "Diffraction-limited storage rings-a window to the science of tomorrow," J. Synchr. Rad. 21, 837-842 (2014).

5. A. Meents et al., "Pink-beam serial crystallography," Nature Comm. 8, 1281 (2017).

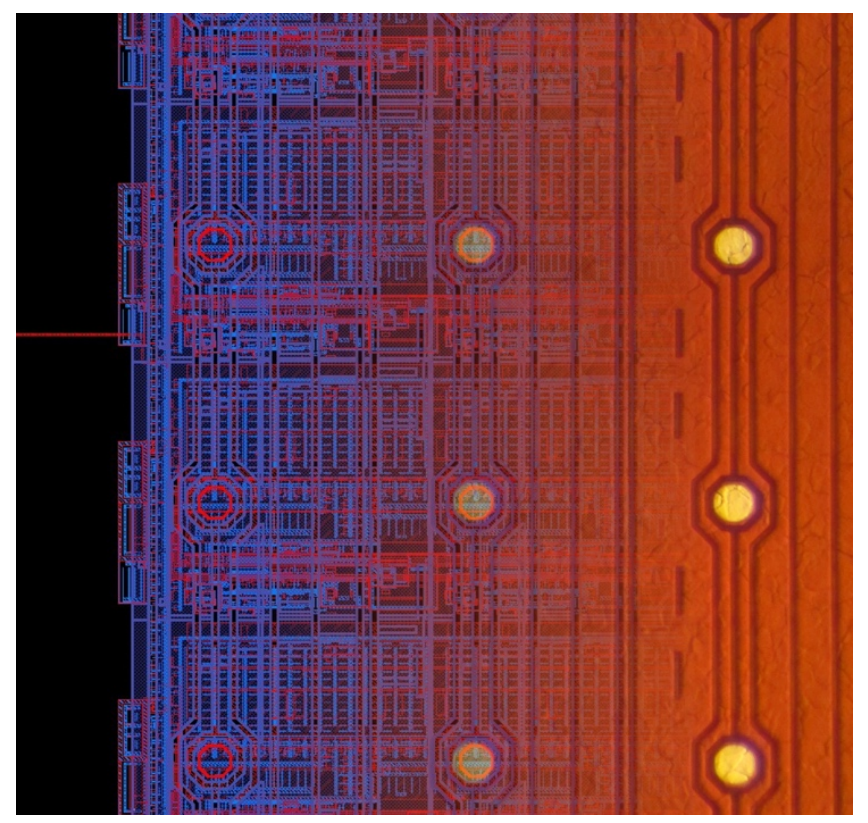

Pixels of the JUNGFRAU detector as seen under a microscope, with an overlay (left) of the pixel structure. The area shows nine of the one million pixels, and the integrated circuit structure that performs the storage and integration of charges that are generated when $\mathrm{X}$-rays are absorbed in the sensor. Image credit: Aldo Mozzanica (PSI). 